\title{
The scale of superpartner masses and electroweakino searches at the high-luminosity LHC
}

\author{
Jia Liu, ${ }^{a, b, c}$ Navin McGinnis, ${ }^{d, e}$ Carlos E.M. Wagner ${ }^{c, e, f}$ and Xiao-Ping Wang ${ }^{e, g}$ \\ ${ }^{a}$ School of Physics and State Key Laboratory of Nuclear Physics and Technology, \\ Peking University, Beijing 100871, China \\ ${ }^{b}$ Center for High Energy Physics, Peking University, \\ Beijing 100871, China \\ ${ }^{c}$ Physics Department and Enrico Fermi Institute, University of Chicago, \\ Chicago, IL 6063\%, U.S.A. \\ ${ }^{d}$ Physics Department, Indiana University, \\ Bloomington, IN 47405, U.S.A. \\ ${ }^{e}$ High Energy Physics Division, Argonne National Laboratory, \\ Argonne, IL 60439, U.S.A. \\ ${ }^{f}$ Kavli Institute for Cosmological Physics, University of Chicago, \\ Chicago, IL 60637, U.S.A. \\ ${ }^{g}$ School of Physics, Beihang University, \\ Beijing 100083, China \\ E-mail: jialiu@pku.edu.cn, nmmcginn@indiana.edu, cwagner@anl.gov, \\ hcwangxiaoping@buaa.edu.cn
}

ABSTRACT: Searches for weakly interacting particles is one of the main goals of the high luminosity LHC run. In this work we study the well motivated cases of electroweakinos with mostly Wino and Bino components. We show the relevance of squark induced t-channel production in defining the production cross section and hence the LHC reach. Moreover, a realistic evaluation of the decay branching ratios show a strong dependence on the sign of $\mu$ and, for negative values of $\mu$, on the relative size of the ratio of $\mu$ to the gaugino masses compared with $\tan \beta$. Overall, unless it is kinematically suppressed, or specific conditions are fulfilled, the Higgs decay channel is the most significant one, and the trilepton channel becomes subdominant with respect to final states including bottom quarks. Although the properties are different than in the Higgsino-Bino case, also in this case the discovery reach extends to mass values that are significantly larger than the ones probed at current luminosities, leading to a strong motivation for the search for electroweakinos in the high luminosity LHC run.

Keywords: Beyond Standard Model, Supersymmetric Standard Model, Higgs Physics ARXIV EPRINT: 2008.11847 


\section{Contents}

1 Introduction 1

2 Mass eigenstates and couplings to $Z$ and SM Higgs 2

2.1 Comments on the anomalous magnetic moment and dark matter for large values of $|\mu|$

3 Production and branching ratios

4 Reach of electroweakino searches according to SUSY scenarios 8

4.1 Current bounds 8

$\begin{array}{lll}4.2 & \text { Future reach and discovery potential } & 11\end{array}$

5 Conclusions

\section{Introduction}

Searches for new particles at the LHC have so far provided no evidence of the existence of new physics at the $\mathrm{TeV}$ scale. These searches are quite sensitive to strongly interacting particles and have excluded the presence of vector like quarks or, in the case of supersymmetry, squarks and gluinos, for masses beyond $1 \mathrm{TeV}$. For examples of recent searches see refs. [1, 2]. It is, however, premature to announce the absence of new physics at the electroweak scale due to these observations. On one hand, these searches have been mostly interpreted within simplified models with simple decay channels designed to maximize the observability of new particles and hence the bounds may be relaxed in the case of more complicated decay channels. More importantly, the searches become mostly insensitive to weakly interacting particles for which the production cross sections become much weaker than the strongly interacting particle ones.

Weakly interacting particles are naturally involved in one of the main hints for physics at the weak scale, namely Dark Matter [3, 4]. The Dark Matter particle appears naturally as part of the weakly interacting sector of extensions of the Standard Model, in a similar way to the appearance of the neutrino in the Standard Model (SM) of particle physics. For heavy particles with masses of the order of the weak scale, the Dark Matter particle is identified with the lightest of these new particles and the stability of these neutral and weakly interacting particles demand the presence of a symmetry, usually discrete (such as R-parity in the MSSM), that forbids the decay of this particles into SM ones. Production of these beyond the SM particles leads to decays into Higgs and weak gauge bosons and the Dark Matter particle which is observed as missing energy.

A particularly well motivated electroweak sector that has been studied in quite detail both theoretically as well as experimentally is the one implied by low-energy supersymmetric extensions, and in particular the one associated to the Minimal Supersymmetry 
Extension of the SM (MSSM) [5-20]. In this case, the electroweak sector consists of two Higgs doublets and their corresponding superpartners (Higgsinos) as well as the superpartners of the weak and hypercharge gauge bosons (Winos and Bino, respectively). The couplings of these particles to the gauge bosons and the Higgs bosons are dictated by the invariance under gauge and supersymmetry transformations, the latter being violated only softly by dimensionful parameters. These mass parameters include the Wino $M_{2}$ and Bino $M_{1}$ masses, as well as the Higgsino mass parameter $\mu$. The Higgs sector is characterized by the mass of the CP-odd Higgs $m_{A}$ and $\tan \beta$, the ratio of the Higgs vacuum expectation values. Due to the supersymmetry relations, the mass of the colored particles also play a role in determining the lightest Higgs mass and also contribute to the t-channel production cross section for gaugino-like particles (the Higginos couple very weakly to the first and second generation quarks.) [21-26].

In a previous article [27], we studied the search for particles in the Higgsino-Bino sector of this model, assuming that the Wino mass is of order of a few $\mathrm{TeV}$ and a decoupled sfermion spectrum. We demonstrated the complementarity of the production of electroweakinos via the heavy Higgs bosons with the ones induced by the direct production of these particles via gauge bosons and, due to the smaller production cross sections, we showed that the regions probed at present are far weaker than the ones that are usually displayed experimentally for the Wino case. Moreover, we showed that the discovery reach of the high luminosity LHC go far beyond the current probed region. The final states including gauge and Higgs bosons played a similarly relevant role in this analysis.

In this work, we extend this analysis to the Wino case, which differs from the Higgsino case in several relevant aspects. On one hand the production cross section has a relevant dependence on the masses of the first and second generation squarks. On the other hand, the branching ratios of the decay of the neutral Winos into Higgs and $Z$ final states depend on the sign of $\mu$, the Higgs decay being in general dominant for positive $\mu$, and also for negative values of $\mu$ unless one is in the proximity of a so-called blind spot solution, that occurs when the ratio of $|\mu|$ to the average gaugino masses is of order $\tan \beta / 2$. This implies a more complex (and weaker) reach for Winos to the one that is usually shown in experimental searches, that rely on large branching ratios and very heavy squark masses.

This work is organized as follows. In section 2, we briefly review the mass eigenstates and mixing for the Wino case and calculate its decay branching ratio to $Z$ and SM Higgs. In section 3, we point out the squark mass dependence for the production cross section and show the parametric dependence of the Wino decay branching ratios on $\mu$ and $\tan \beta$. In section 4 , we show the resultant, current bounds and future reach of the electroweakino searches. We reserve section 5, for our conclusions.

\section{Mass eigenstates and couplings to $Z$ and SM Higgs}

The mass eigenstates and decays modes of all electroweakinos are determined by only four parameters, the Wino and Bino masses $M_{2}$ and $M_{1}$, the Higgsino mass $\mu$, and the ratio of vacuum expectation values of the Higgs doublets $\tan \beta$. The resulting mass matrices for 
the neutralinos and charginos in terms of these parameters are given by

$$
\begin{aligned}
M_{N} & =\left(\begin{array}{cccc}
M_{1} & 0 & -c_{\beta} s_{W} m_{Z} & s_{\beta} s_{W} m_{Z} \\
0 & M_{2} & c_{\beta} c_{W} m_{Z} & -s_{\beta} c_{W} m_{Z} \\
-c_{\beta} s_{W} m_{Z} & c_{\beta} c_{W} m_{Z} & 0 & -\mu \\
s_{\beta} s_{W} m_{Z} & -s_{\beta} c_{W} m_{Z} & -\mu & 0
\end{array}\right), \\
M_{C} & =\left(\begin{array}{cc}
M_{2} & \sqrt{2} s_{\beta} m_{W} \\
\sqrt{2} c_{\beta} m_{W} & \mu
\end{array}\right),
\end{aligned}
$$

where $c_{W}=\cos \theta_{W}, s_{W}=\sin \theta_{W}, m_{Z}$ and $m_{W}$ are the $Z$ and $W$ gauge boson masses, and $\theta_{W}$ is the weak-mixing angle. For further details of the couplings and mass matrices of neutralinos and charginos see, for instance, ref. [9].

The neutralino mass eigenstates are given after diagonlization $Z_{N}^{T} M_{N} Z_{N}=\tilde{m}_{\chi}$ where $\tilde{m}_{\chi}=\operatorname{diag}\left(m_{\chi_{1}^{0}}, m_{\chi_{2}^{0}}, m_{\chi_{3}^{0}}, m_{\chi_{4}^{0}}\right)$ and the mixing matrix, $Z_{N}$, encodes the admixtures of the gauge eigenstates in the neutralinos. In general, the particular form of $Z_{N}$ is not particularly illuminating. However, in this paper we will focus on the case when the Higgsinos are heavy and the low energy spectrum consists of Wino- and Bino-like states. In the limit of $|\mu| \gg M_{1}, M_{2}$, we find for the mixing matrix $Z_{N}$

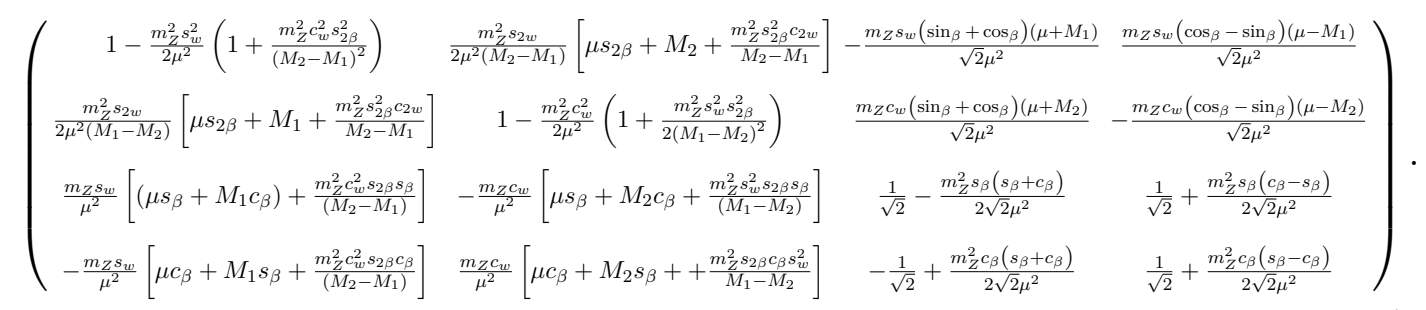

Assuming $M_{2}>M_{1}$, the mass eigenvalues of the NLSP and LSP are then simply approximated by

$$
\begin{aligned}
& m_{\chi_{1}^{0}}=M_{1}-\frac{m_{Z}^{2}}{\mu} s_{w}^{2}\left(\sin _{2 \beta}+\frac{M_{1}}{\mu}\right) \simeq M_{1}, \\
& m_{\chi_{2}^{0}}=M_{2}-\frac{m_{Z}^{2}}{\mu} c_{w}^{2}\left(\sin _{2 \beta}+\frac{M_{2}}{\mu}\right) \simeq M_{2},
\end{aligned}
$$

where for large $|\mu|$ we see that $\chi_{2}^{0}$ and $\chi_{1}^{0}$ are almost pure Wino and Bino mixtures respectively.

The parameters that determine the mass eigenstates and mixing also determine the couplings of electroweakinos to gauge and Higgs bosons through mixing of D-terms. In particular, the couplings of $\chi_{2}^{0}$ to $h / Z$ and $\chi_{1}^{0}, g_{h \chi_{1}^{0} \chi_{2}^{0}}$ and $g_{Z \chi_{1}^{0} \chi_{2}^{0}}$, can be simplified assuming large $\mu,\left|m_{\chi_{1}^{0}}-m_{\chi_{2}^{0}}\right|>m_{h}, m_{Z}$, and the Higgs alignment condition $\alpha \approx \beta-\pi / 2$. We find $^{1}$

$$
\begin{aligned}
& g_{h \chi_{1}^{0} \chi_{2}^{0}}=-\frac{e m_{Z}}{\mu}\left[s_{2 \beta}+\frac{m_{\chi_{1}^{0}}+m_{\chi_{2}^{0}}}{2 \mu}+\frac{m_{Z}^{2} s_{2 \beta}^{2} c_{2 w}}{\mu\left(m_{\chi_{2}^{0}}-m_{\chi_{1}^{0}}\right)}\right], \\
& g_{Z \chi_{1}^{0} \chi_{2}^{0}}=-\frac{e m_{Z}^{2}}{2 \mu^{2}}\left[c_{2 \beta}+\frac{m_{Z}^{2} s_{4 \beta} c_{2 w}}{2 \mu\left(m_{\chi_{2}^{0}}-m_{\chi_{1}^{0}}\right)}\right] .
\end{aligned}
$$

\footnotetext{
${ }^{1}$ Our result in $g_{h \chi_{1}^{0} \chi_{2}^{0}}$ has a minor difference with ref. [28], which omitted $m_{\chi_{1}^{0}}$, probably assuming it to be small.
} 
We see that when $\mu<0$, the Higgs coupling $g_{h \chi_{1}^{0} \chi_{2}^{0}}$ has a blind spot when $s_{2 \beta}+\left(m_{\chi_{1}^{0}}+\right.$ $\left.m_{\chi_{2}^{0}}\right) /(2 \mu) \sim 0$, and thus along this direction the coupling to the Higgs is suppressed and the neutral Wino decays almost exclusively through the Z-channel. Further, for a given pair of neutralino masses the blind spot is determined by the values of $\tan \beta$ and $\mu$. For example, given $m_{\chi_{1}^{0}}+m_{\chi_{2}^{0}}=800 \mathrm{GeV}$, the cancellation happens for $\{\tan \beta, \mu(\mathrm{GeV})\}=\{5,-1040\}$, $\{10,-2020\}$ or $\{20,-4010\}$.

The decay widths of $\chi_{2}^{0} \rightarrow \chi_{1}^{0} h$ and $\chi_{2}^{0} \rightarrow \chi_{1}^{0} Z$ are given by

$$
\begin{aligned}
\Gamma_{h} & =\frac{g_{h \chi_{1}^{0} \chi_{2}^{0}}^{2}}{8 \pi} p_{h} \frac{\left(m_{\chi_{1}^{0}}+m_{\chi_{2}^{0}}\right)^{2}-m_{h}^{2}}{m_{\chi_{2}^{0}}^{2}}, \\
\Gamma_{Z} & =\frac{g_{Z \chi_{1}^{0} \chi_{2}^{0}}^{2}}{8 \pi} p_{Z} \frac{\left(m_{\chi_{1}^{0}}+m_{\chi_{2}^{0}}\right)^{2}-m_{Z}^{2}}{m_{\chi_{2}^{0}}^{2}} \times \frac{\left(m_{\chi_{2}^{0}}-m_{\chi_{1}^{0}}\right)^{2}+2 m_{Z}^{2}}{m_{Z}^{2}},
\end{aligned}
$$

where $p_{h}$ and $p_{Z}$ are the momentum of $h$ and $Z$ in the final state. These results are in agreement with ref. [29]. For a review of electroweakino scenarios in the MSSM and corresponding decay modes see ref. [30].

\subsection{Comments on the anomalous magnetic moment and dark matter for large values of $|\boldsymbol{\mu}|$}

The SM prediction for the muon anomalous magnetic moment, $(g-2)_{\mu}$ differs by about 3.5 standard deviations with respect to the current experimental value measured at the Brookhaven $g-2$ experiment [31-33],

$$
\delta a_{\mu}^{\exp } \simeq(27 \pm 7 \pm 5) \times 10^{-10}
$$

where the errors are associated with experimental and theoretical uncertainties.

The dominant contribution to the muon anomalous magnetic moment in the MSSM [34-39], comes from the chargino induced diagram. This contribution relies on the presence of a relevant Higgsino and Wino component of the light charginos and hence is suppressed when $|\mu|$ becomes sizable. For large values of $|\mu|$, like the ones analyzed in this article, the neutralino contribution may be also relevant. Contrary to Winos, Binos couple to both left- and right-handed muons and tend to provide the most relevant contributions through mixing in the slepton sector, with the parameter $\mu$ providing the necessary left-right slepton mixing. Since this mixing is proportional to $\tan \beta$, sizable neutralino contributions may be obtained for large values of $\mu \tan \beta$ and sleptons that may easily be heavier than the characteristic Wino mass scale discussed in this article. We verified these properties quantitatively by using the code CPsuperH $[40,41]$. As an explicit example, if one takes the extreme values of $\mu=10 \mathrm{TeV}$ and $\tan \beta=50$, considered in this article, values of $M_{1}$ of the order of the weak scale and first and second generation slepton masses of order $700 \mathrm{GeV}$ will be necessary to get the current experimental central value for the muon $g-2$. On the other hand, the third generation sleptons must be heavy in order to avoid problems in the slepton spectrum. For smaller values of $\mu$ and $\tan \beta$, the chargino contributions become relevant. For instance, for $\mu=2 \mathrm{TeV}$ and $\tan \beta=50, M_{2}=400 \mathrm{GeV}$ 

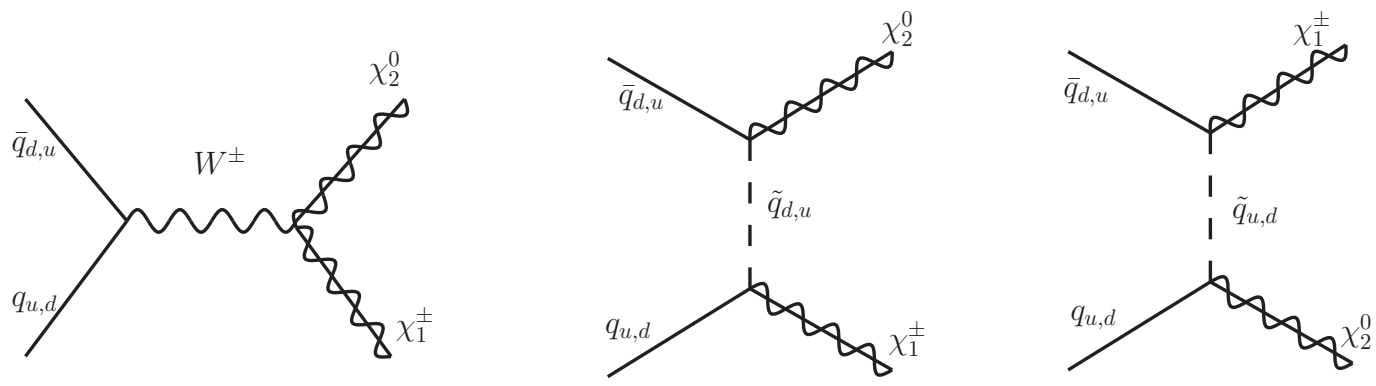

Figure 1. Leading order diagrams contributing to the direct production of electroweakinos at the LHC in the case that the spectrum is Wino-like.

and $M_{1}=250 \mathrm{GeV}$, sleptons with masses of order $450 \mathrm{GeV}$ will lead to the proper $g-2$ contribution, with neutralinos providing about 60 percent of the total contribution.

Dark Matter, on the other hand, may be obtained either by resonant annihilation with scalars or by co-annihilation with the slepton states [3, 4, 42]. For co-annihilation, light sleptons should be close in mass to the Bino states and therefore will lead to additional decays of the heavier Winos into charged and neutral Wino states, which would be in conflict with the heavy scalar assumption of the current work. This can only be avoided in the case of light right-handed sleptons, with small mixing with their left-handed partners. Such small mixing would suppress the neutralino contribution to $(g-2)_{\mu}$ and is difficult to achieve for large values of $|\mu|$ and $\tan \beta$, but is still possible if one assumes a very large hierarchy between the left- and right-slepton masses. Regarding the resonant annihilation via the Higgs states, this may be achieved for moderate values of $\tan \beta$ and values of the Bino mass close to a half of the heavy Higgs masses (the resonant annihilation contribution via the lightest Higgs (or the $Z$ ) is highly suppressed for large values of $\mu$ [43-49] ). Heavy Higgs boson annihilation, on the other hand, will be subject to strong LHC constraints, unless $\tan \beta$ is not very large $[50,51]$. For instance, we verified using MicrOmegas [52] that the proper relic density can be obtained for $\tan \beta=5, \mu=2 \mathrm{TeV}$, and $M_{H} \simeq 600 \mathrm{GeV}^{2}$ provided $M_{1}$ is of order $290 \mathrm{GeV}$, where this value increases to $298 \mathrm{GeV}$ for $\mu=5 \mathrm{TeV}$. The direct detection cross section tends to be suppressed, an order of magnitude or more below the current bounds, due to the large Higgs and $Z$ coupling suppression induced by the large values of $|\mu|$.

\section{Production and branching ratios}

At the LHC, the production of Wino-like electroweakinos, $\chi_{1}^{ \pm}$and $\chi_{2}^{0}$, proceeds mostly through s-channel exchange of a $W$ boson. However, for heavy squarks, the $\chi_{1}^{ \pm}-\chi_{2}^{0}$ pair is subdominantly produced through t-channel exchange of first- and second- generation squarks [21-25], see figure $1 .^{3}$ Apart from the parametric dependence described in the

\footnotetext{
${ }^{2}$ Masses of the heavy Higgs bosons $\gtrsim 500 \mathrm{GeV}$ will have a negligable effect on the discussion of electroweakino branching ratios in the following section.

${ }^{3}$ The same is true for other scenarios such as the Higgsino-Bino scenario. However, in such cases the dependence of the couplings to squarks is proportional to their Yukawa couplings and hence negligible.
} 


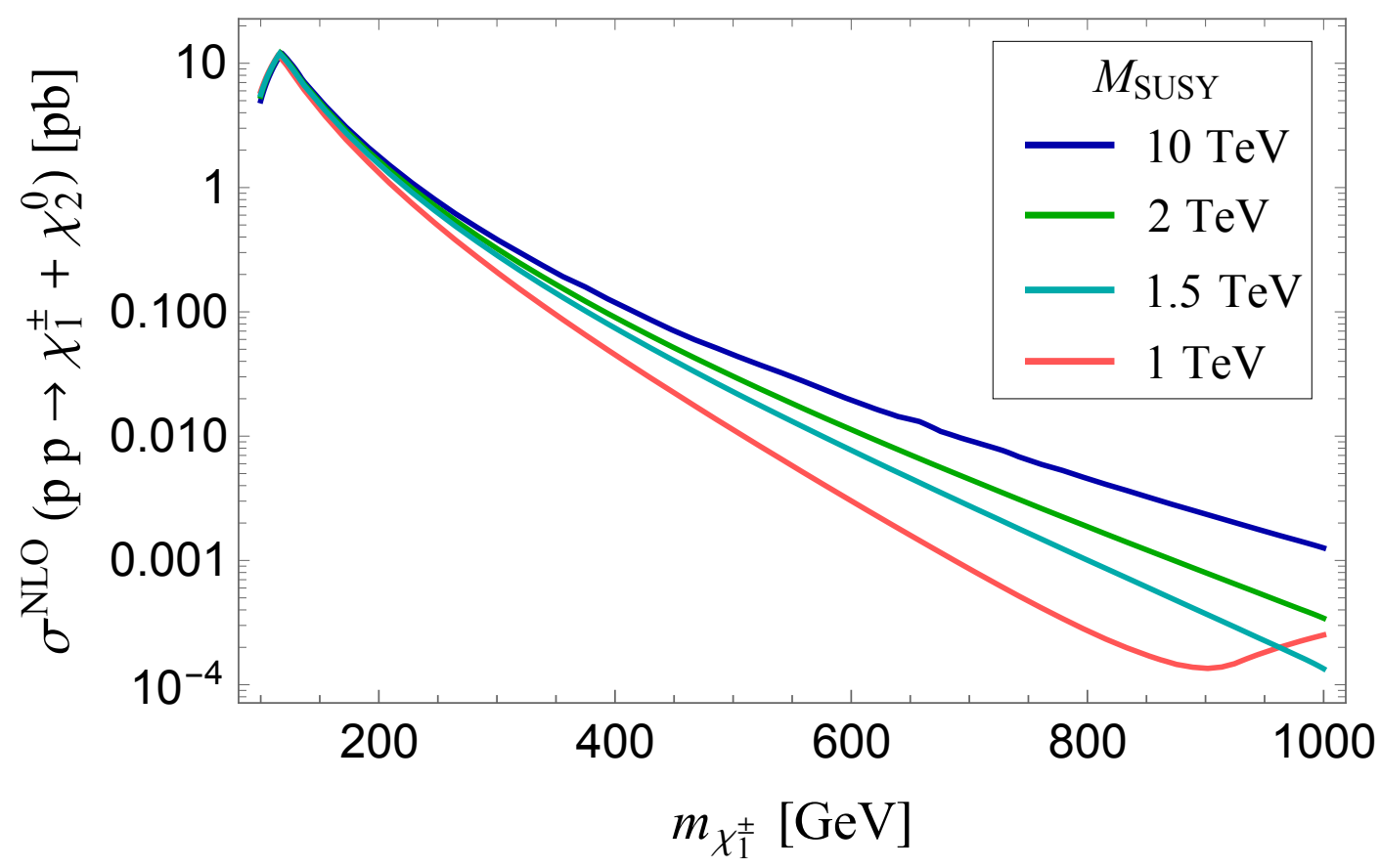

Figure 2. Variation of the Wino-like production cross section for the $13 \mathrm{TeV}$ LHC at NLO when $M_{\mathrm{SUSY}}$ is varied between 1 and $10 \mathrm{TeV} . M_{1}=100 \mathrm{GeV}$ and $\tan \beta=5$ are fixed and $|\mu|=M_{\mathrm{SUSY}}$.

previous section, the overall production modes of $\chi_{1}^{ \pm}$and $\chi_{2}^{0}$ will also have a dependence on the scale of superpartners, $M_{\mathrm{SUSY}}$. The measurement of the Higgs boson mass indicates that stop masses are around $1-10 \mathrm{TeV}$ in the MSSM [53-57]. Further, exclusion of squarks and gluinos have reached well into the $1-2 \mathrm{TeV}$ range [1,2]. Thus, in our discussion we will assume a range of scalar superpartners $M_{\mathrm{SUSY}}=M_{3}=\tilde{m}_{q_{1,2,3}}=\tilde{m}_{l_{1,2,3}}=1-10 \mathrm{TeV}$. For simplicity, we will assume $|\mu|=M_{\text {SUSY }}$ in the main results. However, we will comment on other cases in later sections.

In figure 2, we show the NLO production cross section of Wino-like electroweakinos with respect to the Wino mass for $M_{\mathrm{SUSY}}=|\mu|=1-10 \mathrm{TeV}$. For large Wino masses, the scalar interactions in the production cross section tend to destructively interfere compared to scenarios when superpartners are decoupled well above the weak scale, with the exception of when $M_{\text {SUSY }}=1 \mathrm{TeV}$ for which the mixing of the Wino with the Higgsinos becomes relevant when $M_{2}$ approaches $\mu$. In the range of $m_{\chi_{1}^{ \pm}} \simeq 500-1000 \mathrm{GeV}$, we find that the difference in the production cross section can be close to a factor of $\sim 2-4$. This range of masses is currently in the region of interest of exclusion and/or discovery limits for future searches of electroweakinos at the LHC. Thus, despite being decoupled from the typical searches, the scale of superpartners can have striking consequences on the interpretation of many channels currently being explored.

As discussed in the previous section, the Wino will decay either through a $\mathrm{Z}$ or Higgs boson to $\chi_{1}^{0}$. In the traditional searches, these decay modes are considered to be maximal over the whole range of masses considered. However, as we have pointed out these branching ratios have non-trivial dependence on the same set of parameters that determine the masses 

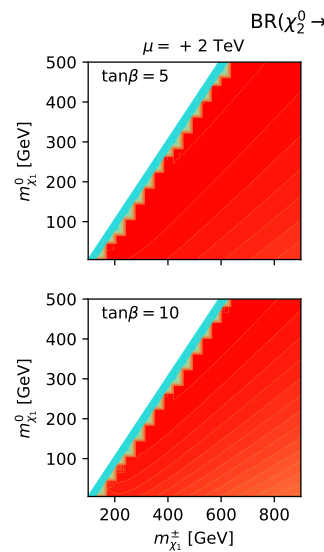
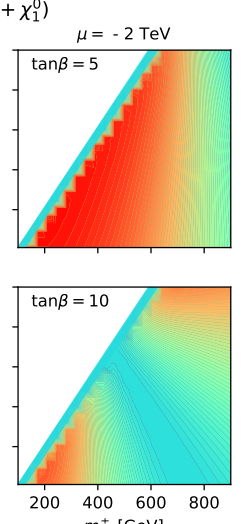

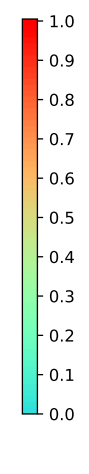

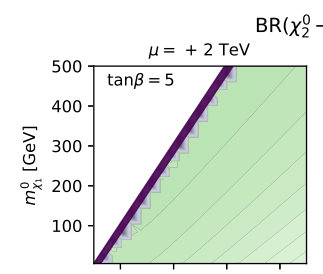

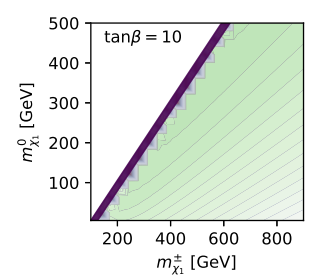

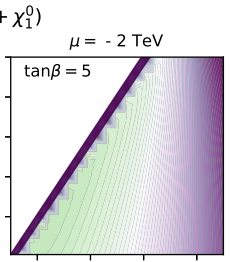

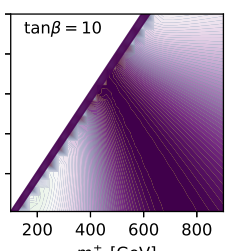

$m_{z_{1}}^{ \pm}[\mathrm{GeV}]$

Figure 3. Left: branching ratio of $\chi_{2}^{0}$ to the SM Higgs boson and $\chi_{1}^{0}$ for $|\mu|=2 \mathrm{TeV}$ and $\tan \beta=$ $5 \& 10$, presented in the $m_{\chi_{1}^{ \pm}}-m_{\chi_{1}^{0}}$ plane. Right: branching ratio of $\chi_{2}^{0}$ to the $\mathrm{Z}$ boson and $\chi_{1}^{0}$ for the same parameters.
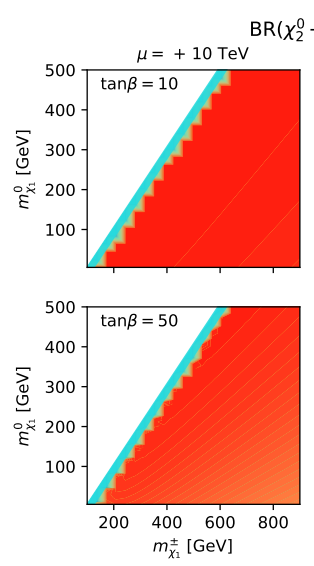

$\mathrm{BR}\left(x_{2}^{0} \rightarrow h+x_{1}^{0}\right)$
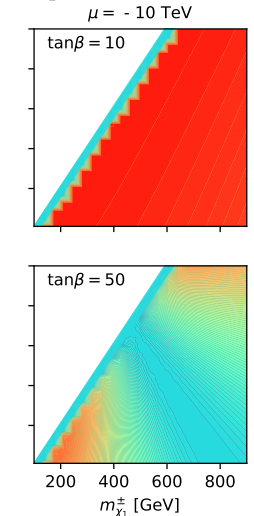
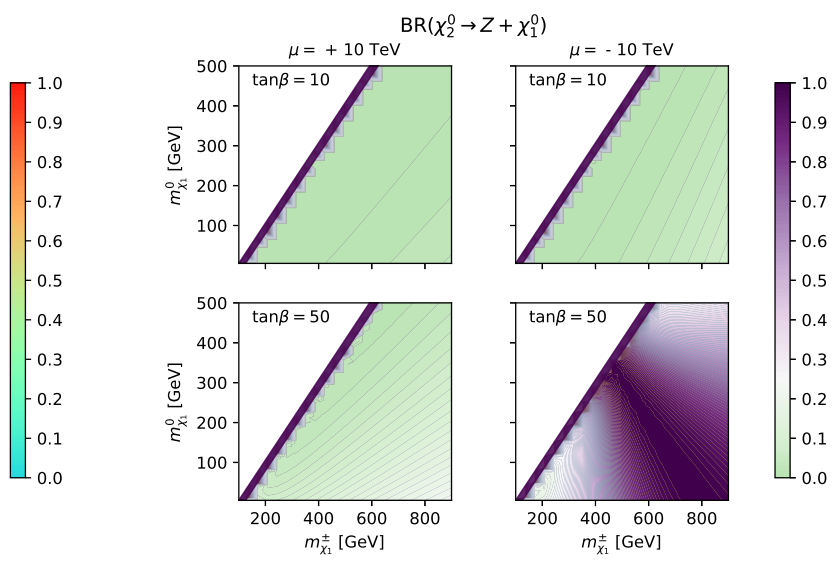

Figure 4. Left: branching ratio of $\chi_{2}^{0}$ to the SM Higgs boson and $\chi_{1}^{0}$ for $|\mu|=10 \mathrm{TeV}$ and $\tan \beta=10 \& 50$, presented in the $m_{\chi_{1}^{ \pm}}-m_{\chi_{1}^{0}}$ plane. Right: branching ratio of $\chi_{2}^{0}$ to the $\mathrm{Z}$ boson and $\chi_{1}^{0}$ for the same parameters.

eigenstates. In figures $3 \& 4$ we show the branching ratios of $\chi_{2}^{0}$ into $Z$ and $h$ for $M_{\text {SUSY }}=$ $2 \& 10 \mathrm{TeV}$ respectively. In each case, we show branching ratios for $\mu= \pm M_{\mathrm{SUSY}}$. For $M_{\mathrm{SUSY}}=2 \mathrm{TeV}$ we show branching ratios for $\tan \beta=5 \& 10$, while for $M_{\mathrm{SUSY}}=10 \mathrm{TeV}$ we take $\tan \beta=10 \& 50$ to show the region of parameters where the blind spot in the Higgs decay is realized. The spectrum and branching ratios are produced using FeynHiggs [57-64] and SUSY-HIT [65], respectively, by scanning $M_{1}=[5,500]$ and $M_{2}=[100,1000]$.

We see that for $M_{\mathrm{SUSY}}=2 \mathrm{TeV}$ the Higgs decay mode is dominant over most of the region of interest. However, for $\mu<0$ and $\tan \beta=10$ we see that the previously discussed blind spot condition may be fulfilled and the $\mathrm{Z}$ decay mode becomes dominant. While for $M_{\mathrm{SUSY}}=10 \mathrm{TeV}$ the Higgs decay mode reaches maximum strength in most of the parameter space whereas the decay of Winos through the $\mathrm{Z}$ boson is negligible everywhere except in the compressed region, $m_{\chi_{2}^{0}} \simeq m_{Z}+m_{\chi_{1}^{0}}$ and for $\mu<0$ and $\tan \beta=50$ in this case. 
We note that such patterns of the branching ratios are not themselves strictly dependent on the scale of the scalar superpartners. Leaving $\mu=-2 \mathrm{TeV}$ fixed and increasing the scale of the scalar superpartners will have the dual effect of increasing the production cross section and leaving the blind spot in a range accessible to, for instance, trilepton searches.

These findings show that, except for particular regions of parameter space, the Higgs decay channel stands out as the most promising decay mode for searches of Wino-like electroweakinos at the LHC. In particular, if $\mu>0$ this decay channel is the only relevant production mode beyond the compressed region, $m_{\chi_{2}^{0}} \simeq m_{Z}+m_{\chi_{1}^{0}}$. This, together with the dependence of the production cross section on masses of the scalar superpartners, gives pertinent information when interpreting current bounds and projecting the future reach of the HL-LHC for electroweakinos.

\section{Reach of electroweakino searches according to SUSY scenarios}

\subsection{Current bounds}

The current reach of electroweakinos at the LHC has been presented in numerous studies by the ATLAS and CMS collaborations refs. [10-14, 16, 17, 19]. For definiteness, in this article we will concentrate on the studies presented by the ATLAS collaboration. The existing searches present bounds on the masses of charginos and neutralinos at the $13 \mathrm{TeV}$ LHC for luminosities ranging from $36 \mathrm{fb}^{-1}$ to $139 \mathrm{fb}^{-1}$ assuming Wino-like crosses sections with superpartners decoupled and maximal branching ratios of $\chi_{2}^{0}$ to either Z or SM Higgs bosons. In this section, we recast the current bounds comparing the reach of electroweakino searches with respect to the scale of superpartners.

The production cross sections and associated branching ratios are obtained over the Wino-Bino parameter space, $M_{1}=[5,500]$ and $M_{2}=[100,1000]$, using and Prospino-2.1 [66] and SUSY-HIT respectively. For each point in the scan, we compare the result with the experimental upper limits on the cross section for decays leading to trilepton or $h \rightarrow b \bar{b}$ channels $[14,16,17,19]$,

$$
p p \rightarrow \chi_{1}^{ \pm}+\chi_{2}^{0} \rightarrow W^{ \pm}+Z / h+\mathbb{E}_{T}= \begin{cases}3 \ell+\mathbb{E}_{T} & \text { for } Z \rightarrow \ell \ell \\ 1(0) \ell+b \bar{b}+\mathbb{E}_{T} & \text { for } h \rightarrow b \bar{b} .\end{cases}
$$

In this and subsequent sections, we focus mainly on two scenarios when $M_{\mathrm{SUSY}}=$ $|\mu|=2 \mathrm{TeV}$ and $M_{\mathrm{SUSY}}=|\mu|=10 \mathrm{TeV} \cdot{ }^{4}$ As discussed in the previous section, for a given $|\mu|$ the blind spot in the Higgs decay occurs for different regions of $\tan \beta$. Thus, in order to present both the worst and best case scenario for the reach of the searches we consider $\tan \beta=5 \& 10$ when $M_{\mathrm{SUSY}}=|\mu|=2 \mathrm{TeV}$, and $\tan \beta=10 \& 50$ when $M_{\mathrm{SUSY}}=|\mu|=10 \mathrm{TeV}$. In each case, since we are recasting the current bounds taken from the data available on HEP-data, we do not extend our results beyond what has already been explored by the experimental collaborations as such an analysis would require an artificial presentation of signal and background efficiencies which would typically improve with increased data. Thus, we consider our results conservative in this sense.

\footnotetext{
${ }^{4}$ Lower squark masses $1 \mathrm{TeV}$ would result in abysmal reach for electroweakinos as the interference in the production cross section is maximal.
} 

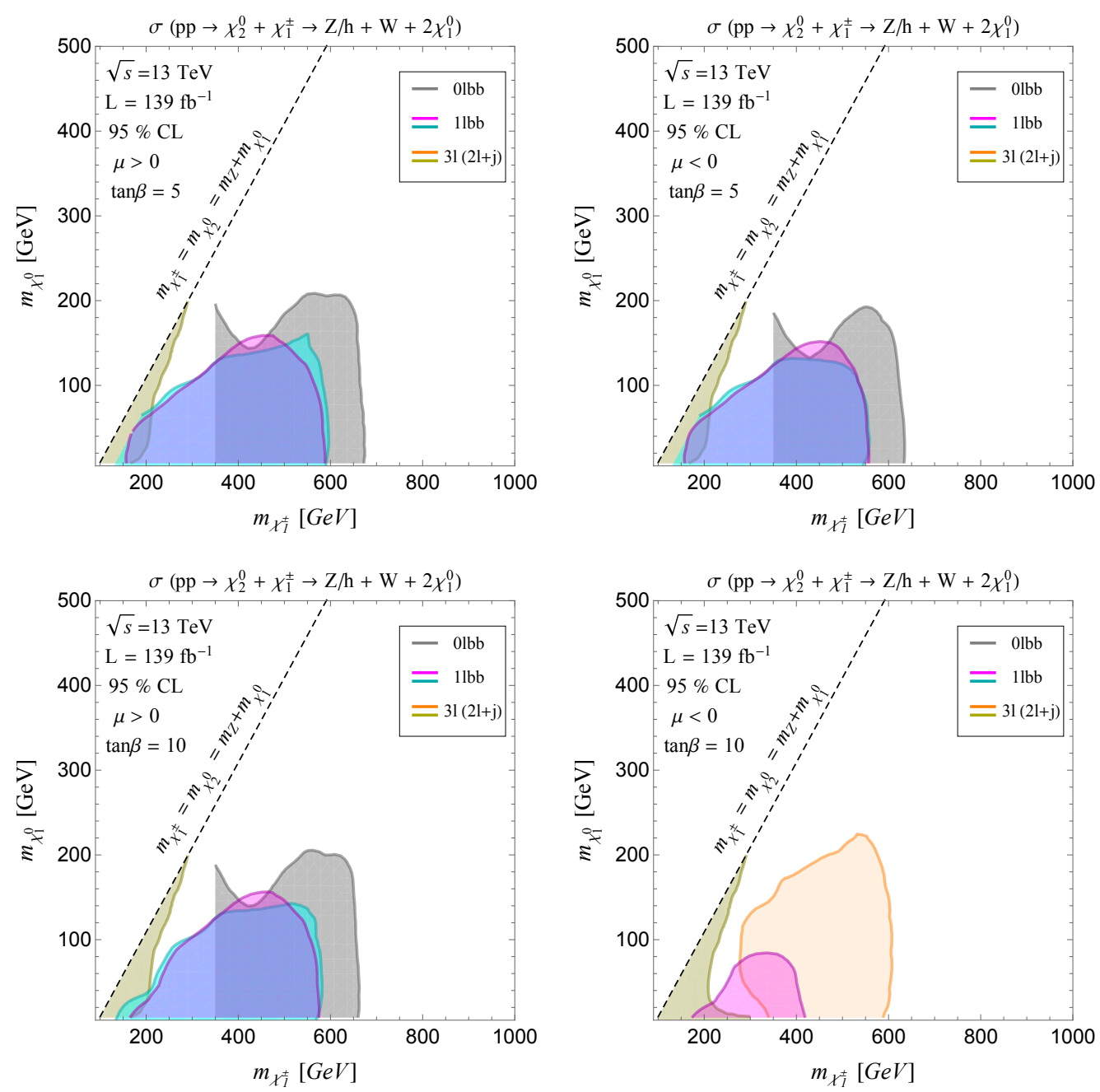

Figure 5. 95\% confidence level bounds on the Wino-Bino scenario projected to integrated luminosity $L=139 \mathrm{fb}^{-1}$. In the top (bottom) panel, we show the bounds for $\tan \beta=5(\tan \beta=10)$ and $M_{\mathrm{SUSY}}=|\mu|=2 \mathrm{TeV}$. The $0 \ell b b$ (gray) [16] and $1 \ell b b$ (magenta, cyan) $[16,17]$ bounds are projected from searches of the $\chi_{2}^{0} \chi_{1}^{ \pm} \rightarrow h W+2 \chi_{1}^{0}$ channel, with $h \rightarrow \bar{b} b$ and $W$ decay to hadronic or leptonic final states. The $3 \ell$ (dark yellow) [19] and $3 \ell / 2 \ell+\mathrm{j}$ (orange) [15] bounds are projected from the $\chi_{2}^{0} \chi_{1}^{ \pm} \rightarrow Z W+2 \chi_{1}^{0}$ channel, with $Z \rightarrow 2 \ell$.

In figure 5 , we show the result of recasting the current bounds for $M_{\mathrm{SUSY}}=|\mu|=$ $2 \mathrm{TeV}$. The gray and magenta shaded regions show the bounds from a search of the decay $\chi_{2}^{0} \chi_{1}^{ \pm} \rightarrow h W+2 \chi_{1}^{0}$, followed by $h \rightarrow b \bar{b}$, in final states with a pair $b$-quarks and zero or one leptons respectively [16]. We have denoted these channels as $0 \ell b b$ and $1 \ell b b$. The cyan region shows the corresponding bound from an independent search of the $1 \ell b b$ channel [17]. The orange shaded region, denoted as $3 \ell(2 \ell+j)$, shows our recasting of the bounds resulting from a search of the $\chi_{2}^{0} \chi_{1}^{ \pm} \rightarrow Z W+2 \chi_{1}^{0}$ decay, followed by $Z \rightarrow \ell^{+} \ell^{-}$, in the statistical combination of final states with 3 leptons and 2 leptons plus jets [14]. Finally, the dark yellow region shows the bounds from a similar trilepton search focused in the compressed electroweakino spectrum [19]. 

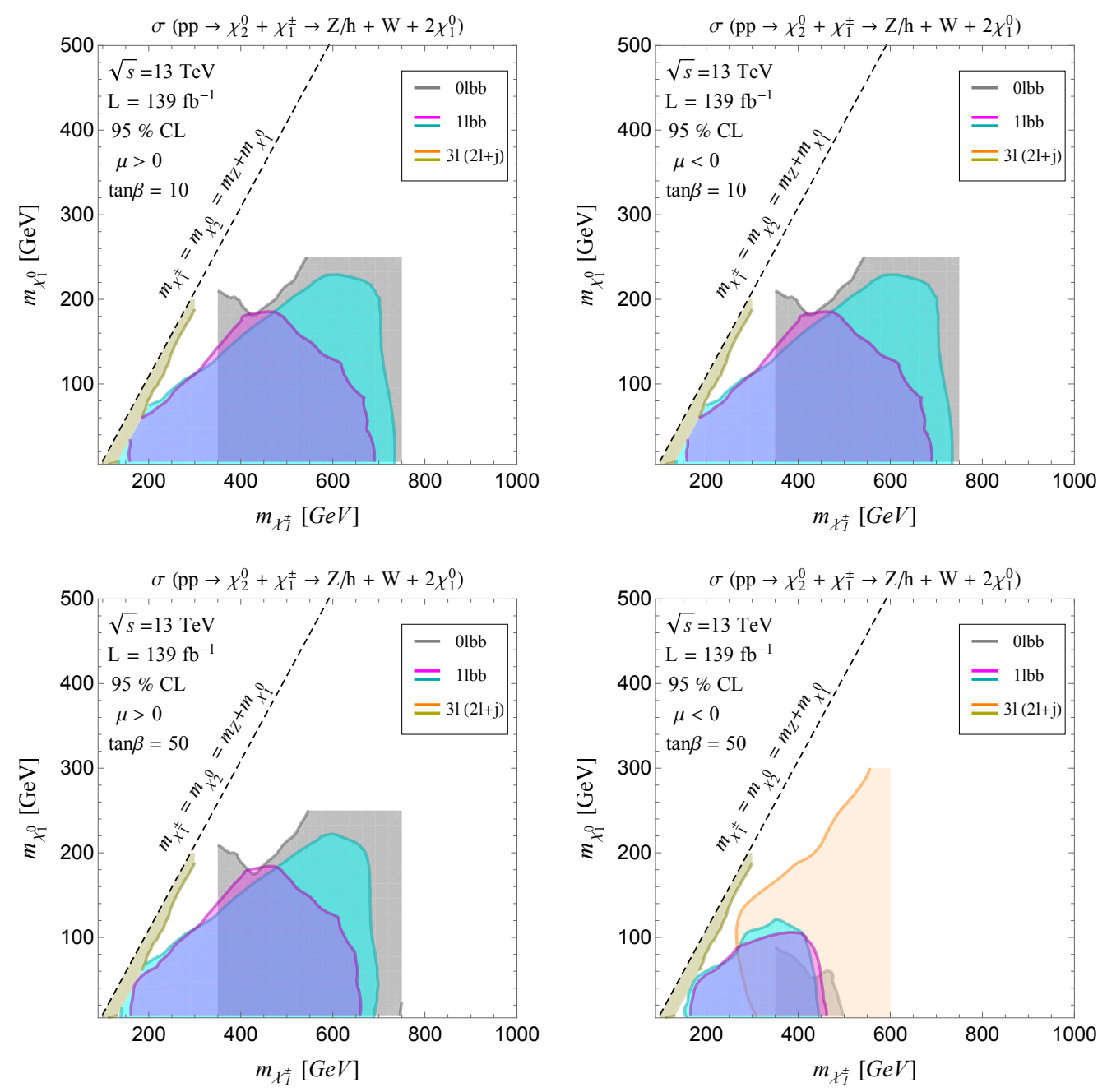

Figure 6. 95\% confidence level bounds on the Wino-Bino scenario projected for integrated luminosity of $L=139 \mathrm{fb}^{-1}$ with $M_{\mathrm{SUSY}}=|\mu|=10 \mathrm{TeV}$. The labels are similar to figure 5 .

Due to the decrease in the production cross section, the resulting bounds from the Higgs channel are weaker than what is typically presented, reaching slightly above chargino masses $m_{\chi_{1}^{ \pm}} \simeq 600 \mathrm{GeV}$ and neutralino masses $m_{\chi_{1}^{ \pm}} \simeq 200 \mathrm{GeV}$. Meanwhile the trilepton searches lose sensitivity almost over the whole range of masses, except in the region of parameters where the blind spot appears in the Higgs decay mode, see figure 3. In contrast, when $M_{\text {SUSY }}=|\mu|=10 \mathrm{TeV}$, figure 6 , we find similar reach in the Higgs channel as is currently expected (in this and subsequent figures, regions of parameters that were left unexplored by the experimental analyses are shown at the edge of the bounds by sharp edges without solid lines). The trilpeton searches again lose sensitivity everywhere beyond the compressed region, and except when $\mu<0$ and $\tan \beta=50$ due to the suppression of the branching ratio of $\chi_{2}^{0}$ to $Z$. However, in this case the overall reach also improves due to the increase in the production cross section.

The assumption that $M_{\mathrm{SUSY}}=|\mu|$ places strong constraints on both the production cross section, patterns of decays for the lightest electroweakino states, and thus the resulting 

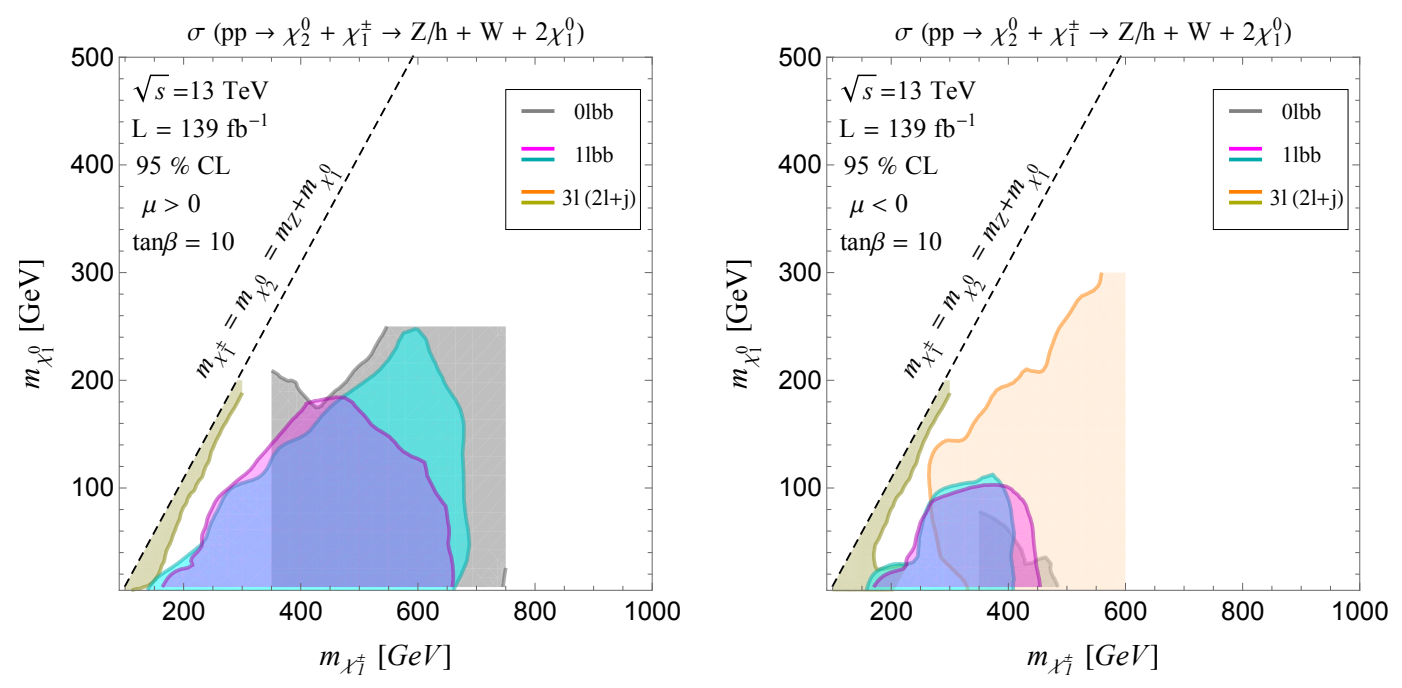

Figure 7. 95\% confidence level bounds on the Wino-Bino scenario projected to integrated luminosity $L=139 \mathrm{fb}^{-1}$, assuming $\tan \beta=10,|\mu|=2 \mathrm{TeV}$, and $M_{\mathrm{SUSY}}=10 \mathrm{TeV}$. The labels are similar to figure 5 .

bounds. Other scenarios with different hierarchies, such as in Split Supersymmetry [67-69] where sfermions are much heavier than the gauginos, are also well motivated. In figure 7 we show the bounds for $\tan \beta=10,|\mu|=2 \mathrm{TeV}$, and $M_{\mathrm{SUSY}}=10 \mathrm{TeV}$. The overall effect on the bounds is twofold. The decoupling of scalars gives an increase in the production cross section yeilding a slightly larger reach in both the Higgs and trilepton channels, and for negative $\mu$ the trilepton searches remain sensitive to the blind spot resulting in an even larger reach compared to the case of a universal SUSY scale (bottom right panel of figure 5).

\subsection{Future reach and discovery potential}

In this section, we assess the ultimate reach and discovery potential of Wino-like electroweakinos at the HL-LHC. As in the previous section, we show the projected bounds resulting from the dependence of the scale of superpartners, $|\mu|$, and $\tan \beta$. Throughout this section, we stress the ultimate reach of electroweakino searches with respect to the squark masses. The current bounds shown in the previous section suggest electroweakino masses well above $100 \mathrm{GeV}$. From figure 2 it is clear that in this region of parameters, squarks with masses of $10 \mathrm{TeV}$ can boost the Wino cross section by close to a factor of 2 compared to $2 \mathrm{TeV}$. Thus, in this section we project the bounds and discovery potential of electroweakinos for the HL-LHC for $M_{\mathrm{SUSY}}=2$ and $10 \mathrm{TeV}$. The lower bound, $M_{\mathrm{SUSY}}=2 \mathrm{TeV}$, is chosen to conservatively satisfy current bounds on squark masses, while $M_{\mathrm{SUSY}}=10 \mathrm{TeV}$ gives the maximum boost to the electroweakino production cross section leading to the strongest potential reach for these particles. For other theoretical projections of electroweakino searches in the (N)MSSM see [26, 44, 70-73].

In figure 8 , we show the projected bounds for integrated luminosity of $3 \mathrm{ab}^{-1}$ with $M_{\mathrm{SUSY}}=|\mu|=2 \mathrm{TeV}$ and $\tan \beta=5$ (10) in the top (bottom) panels. As before, the Higgs 

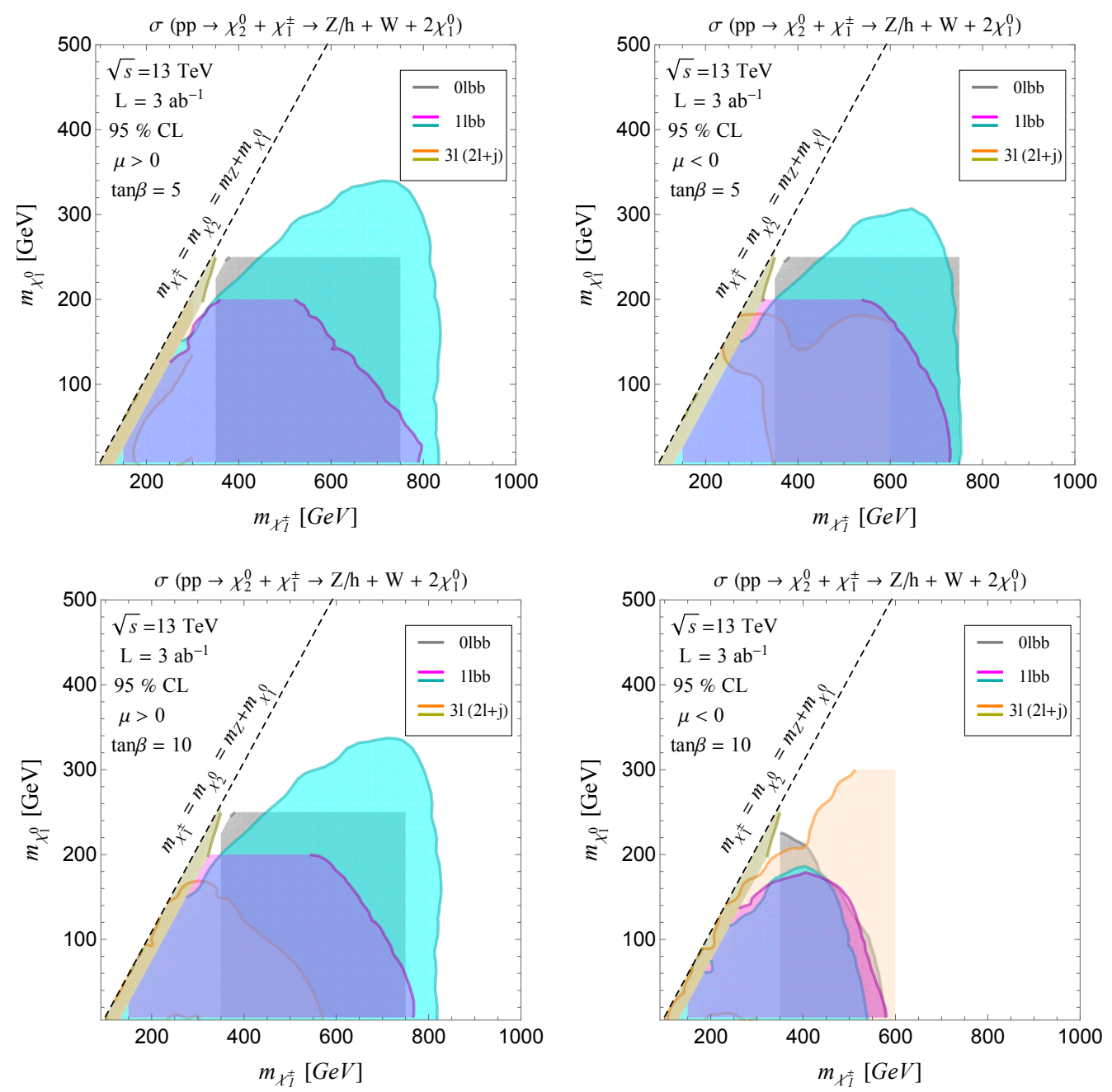

Figure 8. 95\% confidence level bounds on the Wino-Bino scenario projected for integrated luminosity of $L=3 \mathrm{ab}^{-1}$ with $M_{\mathrm{SUSY}}=|\mu|=2 \mathrm{TeV}$. The labels are similar to figure 5 .

decay channel remains the dominant search channel for most of the range of chargino and neutralino masses, with the ultimate reach extending the bound for $m_{\chi_{1}^{ \pm}}$beyond $850 \mathrm{GeV}$ and $m_{\chi_{1}^{0}}$ to almost $400 \mathrm{GeV}$ when $\mu>0$ and $\tan \beta=5$. However, we see again that these conclusions differ significantly when the coupling of $\chi_{2}^{0}$ to the SM Higgs crosses the blind spot. In this case, the trilepton channel dominates covering a similar range of masses. In figure 9 , we show the $5-\sigma$ discovery regions for the same set of parameters. Comparing each panel to the respective bound in figure 5 we see that there is a significant region of masses in the discovery region at the HL-LHC not excluded by the current searches. Such a region includes $m_{\chi_{1}^{ \pm}} \gtrsim 600 \mathrm{GeV}, m_{\chi_{1}^{0}} \gtrsim 200 \mathrm{GeV}$, and a far better coverage of the compressed region $m_{\chi_{1}^{ \pm}}-m_{\chi_{1}^{0}} \simeq m_{Z}$.

When $M_{\text {SUSY }}=10 \mathrm{TeV}$ the production cross section reaches maximal values over the whole range of masses giving the strongest expected reach at the HL-LHC. In figure 10 and figure 11, we show the corresponding 95\% CL and 5- $\sigma$ discovery bounds with $M_{\mathrm{SUSY}}=|\mu|$. Here the $95 \%$ CL bounds on chargino masses from Higgs decay searches begin to reach the 

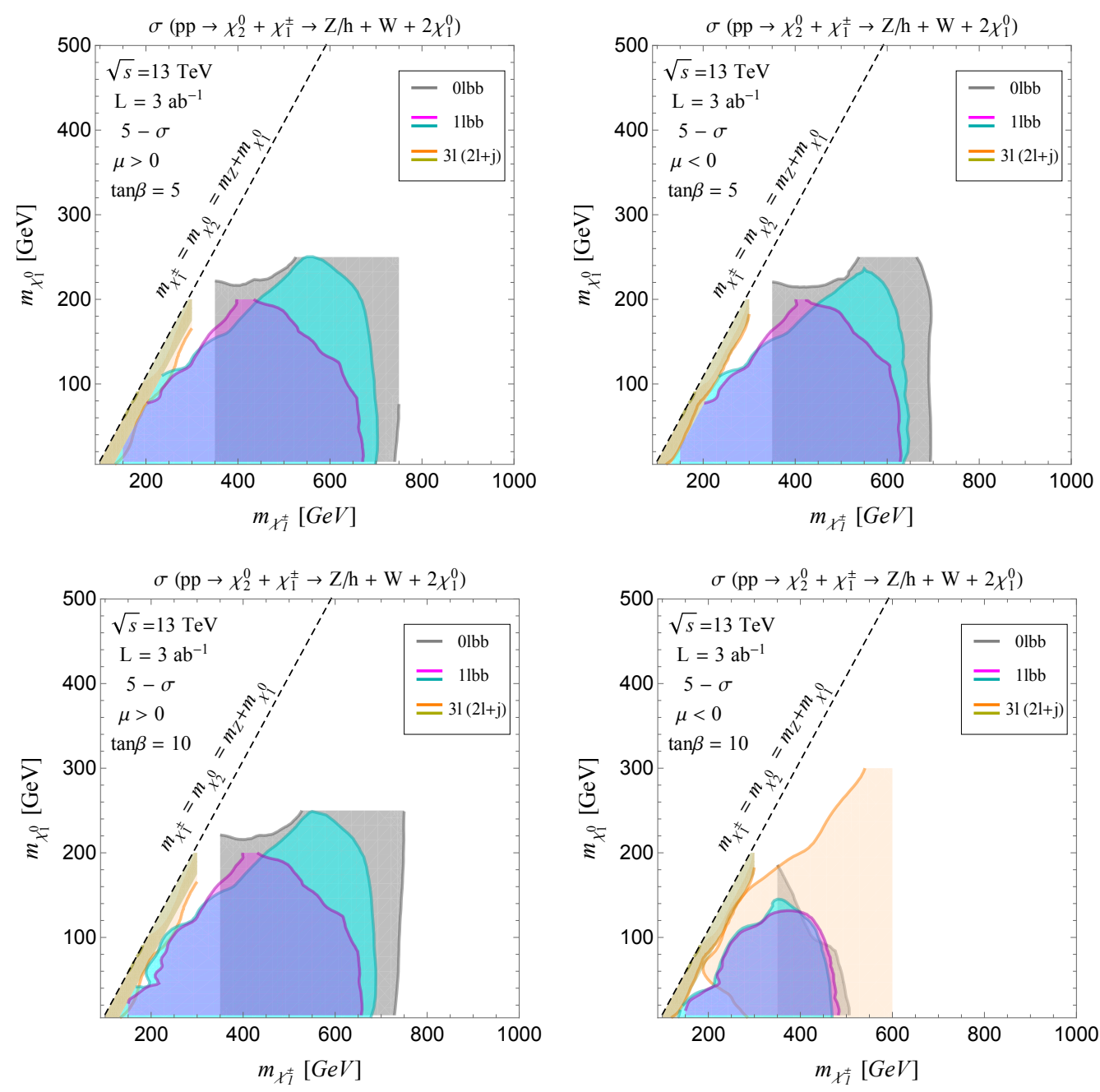

Figure 9. The future potential on the Bino-Wino scenario projected for $5 \sigma$ reach at $3 \mathrm{ab}^{-1}$ with $M_{\mathrm{SUSY}}=|\mu|=2 \mathrm{TeV}$. The labels are similar to figure 5 .

$\mathrm{TeV}$ scale and beyond $400 \mathrm{GeV}$ for neutralinos. In this case, the discovery region extends to $m_{\chi_{1}^{ \pm}} \gtrsim 750 \mathrm{GeV}$ and $m_{\chi_{1}^{0}} \gtrsim 250 \mathrm{GeV}$, and is significantly stronger than the current bounds, shown in figure 6, implying again a strong discovery potential at the HL-LHC run.

\section{Conclusions}

The search for electroweak interacting particles is, together with precision measurements of the Higgs couplings, one of the most promising activities in the HL-LHC era. In this article, we critically reanalyzed the search for electroweakinos in the case of a Higgsino mass parameter significantly larger than the weak scale. We showed that the signatures of Wino production depend crucially on three parameters: the first and second generation squark masses, which control the t-channel contribution to the Wino production cross section, the sign of $\mu$, which control the mixing parameter determining the decay of the neutral Winos into $Z$ or $h$ final states, and finally the relative size of the ratio of the Higgsino mass 

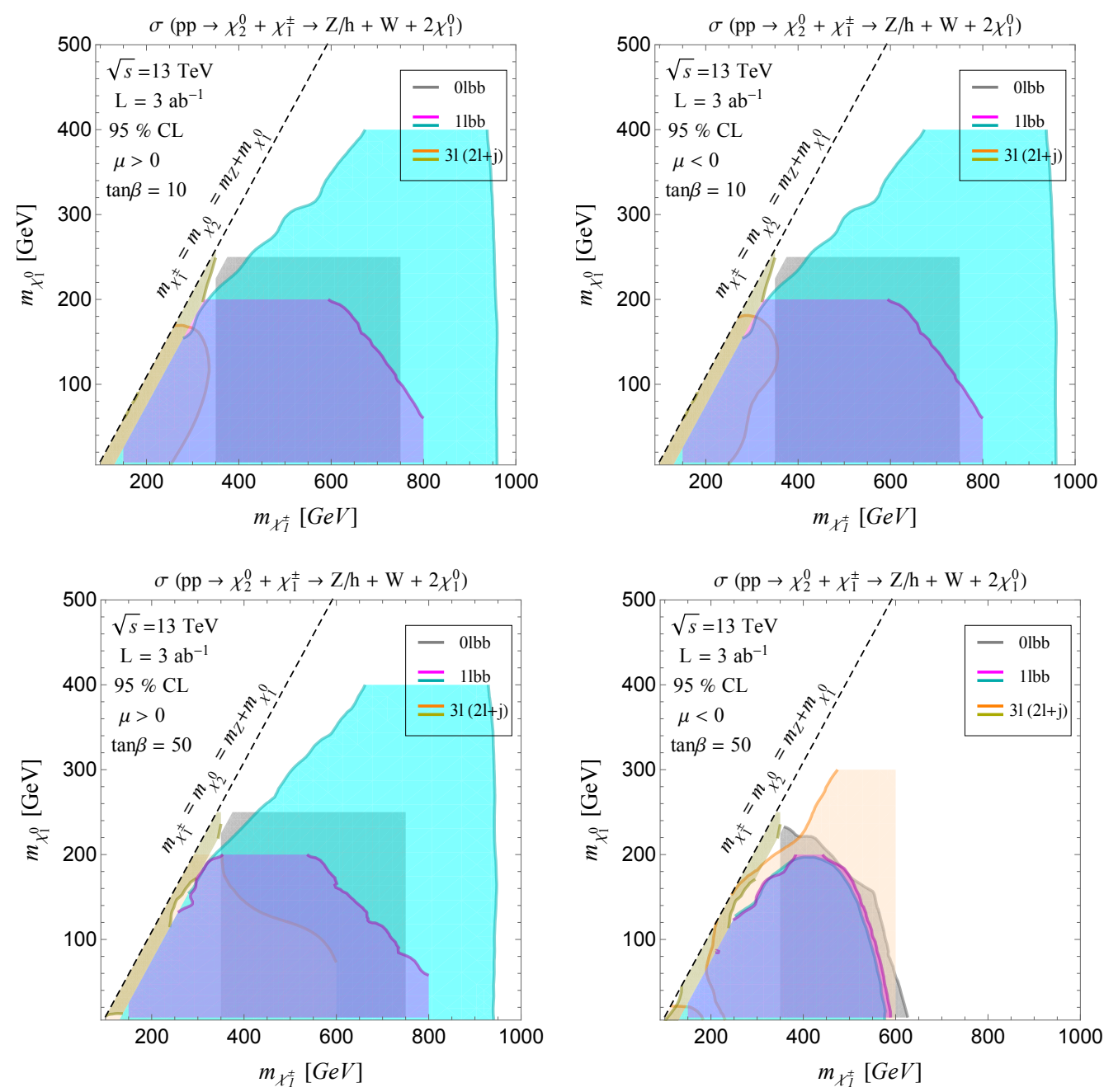

Figure 10. Constraints on the Bino-Wino scenario projected at $3 \mathrm{ab}^{-1}$ at $95 \%$ confidence level with $M_{\mathrm{SUSY}}=|\mu|=10 \mathrm{TeV}$. The labels are similar to figure 5 .

parameter to the average gaugino mass to $\tan \beta$, which control the proximity to the blind spot for the decay of the neutral Wino into Higgs states for negative values of $\mu$.

The t-channel contribution to the cross section interferes destructively with the schannel one and hence the cross section becomes larger for larger squark masses. This destructive interference is still sizable for squark masses of the order of $2 \mathrm{TeV}$, but becomes weak for squark masses above the $5 \mathrm{TeV}$ scale, for which the maximal reach is therefore achieved. These very large values of the squark masses are implicitly assumed in the experimental presentation of the LHC bounds for Wino-like particles decaying into lighter Bino states. It is important to stress that such dependence is not present in the production of Higgsino states, which couple with the first and second generation squarks via their small Yukawa couplings. We refer to ref. [27] for the Higgsino search analysis.

In general, the Higgs decay mode provides the dominant decay branching ratio of the neutral Winos and hence the tri-lepton channel looses significance unless the mass difference between the neutral Winos and Binos are below the Higgs mass scale or one is in 

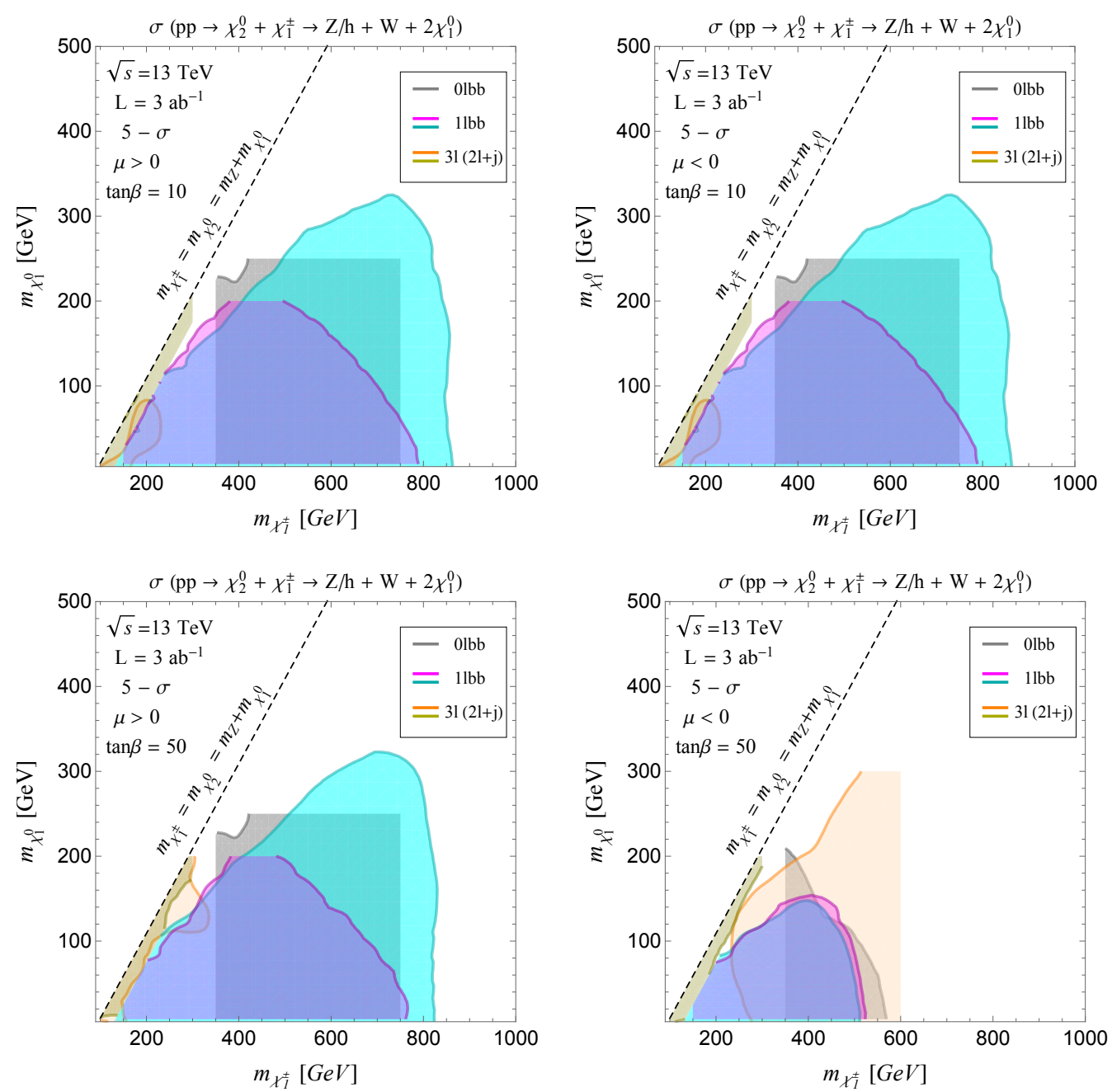

Figure 11. The future potential on the Bino-Wino scenario projected for $5 \sigma$ reach at $3 \mathrm{ab}^{-1}$ with $M_{\mathrm{SUSY}}=|\mu|=10 \mathrm{TeV}$. The labels are similar to figure 5 .

the proximity of the previously mentioned blind spot. For positive values of $\mu$ with respect to the average gaugino masses and large mass differences, the Branching ratio of the Higgs decay is very close to one. The blind spot only occurs for negative values of $\mu$, in which case there may be a rich interplay between the Higgs decay and $Z$ decay searches.

Two relevant conclusions of this study is that, depending on the parameters, the current exclusions limits may be significantly weaker than the ones displayed in experimental searches and, most importantly, the discovery reach of the HL-LHC greatly exceeds the region probed at current luminosities. This, together with similar results obtained in the case of Higgsino searches [27], provides a strong motivation for the future electroweakino searches in the high luminosity LHC era.

\section{Acknowledgments}

Work at University of Chicago is supported in part by U.S. Department of Energy grant number DE-FG02-13ER41958. Work at ANL is supported in part by the U.S. Depart- 
ment of Energy under Contract No. DE-AC02-06CH11357. NM acknowledges support by the U.S. Department of Energy, Office of Science, Office of Work- force Development for Teachers and Scientists, Office of Science Graduate Student Research (SCGSR) program. The SCGSR program is administered by the Oak Ridge Institute for Science and Education (ORISE) for the DOE. ORISE is managed by ORAU under contract number de-sc0014664. The work of JL is supported by National Science Foundation of China under Grant No. 12075005 and by Peking University under startup Grant No. 7101502458. The work of XPW is supported by National Science Foundation of China under Grant No. 12005009.

Open Access. This article is distributed under the terms of the Creative Commons Attribution License (CC-BY 4.0), which permits any use, distribution and reproduction in any medium, provided the original author(s) and source are credited.

\section{References}

[1] ATLAS collaboration, Search for squarks and gluinos in final states with jets and missing transverse momentum using $36 \mathrm{fb}^{-1}$ of $\sqrt{s}=13 \mathrm{TeV}$ pp collision data with the ATLAS detector, Phys. Rev. D 97 (2018) 112001 [arXiv:1712.02332] [INSPIRE].

[2] CMS collaboration, Search for natural and split supersymmetry in proton-proton collisions at $\sqrt{s}=13 \mathrm{TeV}$ in final states with jets and missing transverse momentum, JHEP 05 (2018) 025 [arXiv: 1802.02110] [INSPIRE].

[3] G. Jungman, M. Kamionkowski and K. Griest, Supersymmetric dark matter, Phys. Rept. 267 (1996) 195 [hep-ph/9506380] [INSPIRE].

[4] G. Bertone, D. Hooper and J. Silk, Particle dark matter: evidence, candidates and constraints, Phys. Rept. 405 (2005) 279 [hep-ph/0404175] [INSPIRE].

[5] P. Fayet, Supersymmetry and weak, electromagnetic and strong interactions, Phys. Lett. B 64 (1976) 159 [INSPIRE].

[6] P. Fayet, Spontaneously broken supersymmetric theories of weak, electromagnetic and strong interactions, Phys. Lett. B 69 (1977) 489 [INSPIRE].

[7] J.F. Gunion et al., Calculation and phenomenology of two-body decays of neutralinos and charginos to W, Z, and Higgs bosons, Int. J. Mod. Phys. A 2 (1987) 1145 [inSPIRE].

[8] H.E. Haber and G.L. Kane, The search for supersymmetry: probing physics beyond the standard model, Phys. Rept. 117 (1985) 75 [INSPIRE].

[9] S.P. Martin, A Supersymmetry primer, Adv. Ser. Direct. High Energy Phys. 21 (2010) 1 [hep-ph/9709356] [INSPIRE].

[10] CMS collaboration, Search for electroweak production of charginos and neutralinos in WH events in proton-proton collisions at $\sqrt{s}=13$ TeV, JHEP 11 (2017) 029 [arXiv:1706.09933] [INSPIRE].

[11] CMS collaboration, Search for electroweak production of charginos and neutralinos in multilepton final states in proton-proton collisions at $\sqrt{s}=13 \mathrm{TeV}$, JHEP 03 (2018) 166 [arXiv: 1709.05406] [INSPIRE].

[12] CMS collaboration, Combined search for electroweak production of charginos and neutralinos in proton-proton collisions at $\sqrt{s}=13 \mathrm{TeV}$, JHEP 03 (2018) 160 [arXiv:1801.03957] [INSPIRE]. 
[13] CMS collaboration, Search for new physics in events with two soft oppositely charged leptons and missing transverse momentum in proton-proton collisions at $\sqrt{s}=13$ TeV, Phys. Lett. B 782 (2018) 440 [arXiv: 1801.01846] [INSPIRE].

[14] ATLAS collaboration, Search for electroweak production of supersymmetric particles in final states with two or three leptons at $\sqrt{s}=13 \mathrm{TeV}$ with the ATLAS detector, Eur. Phys. J. C 78 (2018) 995 [arXiv: 1803.02762] [INSPIRE].

[15] ATLAS collaboration, Search for chargino-neutralino production using recursive jigsaw reconstruction in final states with two or three charged leptons in proton-proton collisions at $\sqrt{s}=13$ TeV with the ATLAS detector, Phys. Rev. D 98 (2018) 092012 [arXiv:1806.02293] [INSPIRE].

[16] ATLAS collaboration, Search for chargino and neutralino production in final states with a Higgs boson and missing transverse momentum at $\sqrt{s}=13 \mathrm{TeV}$ with the ATLAS detector, Phys. Rev. D 100 (2019) 012006 [arXiv: 1812.09432] [INSPIRE].

[17] ATLAS collaboration, Search for direct production of electroweakinos in final states with one lepton, missing transverse momentum and a Higgs boson decaying into two b-jets in pp collisions at $\sqrt{s}=13$ TeV with the ATLAS detector, Eur. Phys. J. C 80 (2020) 691 [arXiv: 1909.09226] [INSPIRE].

[18] ATLAS collaboration, Search for electroweak production of charginos and sleptons decaying in final states with two leptons and missing transverse momentum in $\sqrt{s}=13$ TeV pp collisions using the ATLAS detector, ATLAS-CONF-2019-008 (2019).

[19] ATLAS collaboration, Search for chargino-neutralino production with mass splittings near the electroweak scale in three-lepton final states in $\sqrt{s}=13$ TeV pp collisions with the ATLAS detector, ATLAS-CONF-2019-020 (2019).

[20] ATLAS collaboration, Search for direct production of electroweakinos in final states with missing transverse momentum and a Higgs boson decaying into photons in pp collisions at $\sqrt{s}=13$ TeV with the ATLAS detector, JHEP 10 (2020) 005 [arXiv: 2004.10894] [INSPIRE].

[21] H. Baer, C. Kao and X. Tata, Aspects of chargino-neutralino production at the Tevatron collider, Phys. Rev. D 48 (1993) 5175 [hep-ph/9307347] [INSPIRE].

[22] H. Baer, C.-h. Chen, F. Paige and X. Tata, Trileptons from chargino-neutralino production at the CERN Large Hadron Collider, Phys. Rev. D 50 (1994) 4508 [hep-ph/9404212] [INSPIRE].

[23] J.L. Lopez, D.V. Nanopoulos, X. Wang and A. Zichichi, Supersymmetry dileptons and trileptons at the Tevatron, Phys. Rev. D 52 (1995) 142 [hep-ph/9412346] [INSPIRE].

[24] H. Baer, C.-h. Chen, F. Paige and X. Tata, Signals for minimal supergravity at the CERN large hadron collider: multi-jet plus missing energy channel, Phys. Rev. D 52 (1995) 2746 [hep-ph/9503271] [INSPIRE].

[25] H. Baer, C.-h. Chen, C. Kao and X. Tata, Supersymmetry reach of an upgraded Tevatron collider, Phys. Rev. D 52 (1995) 1565 [hep-ph/9504234] [INSPIRE].

[26] F. Yu, Anatomizing exotic production of the Higgs boson, Phys. Rev. D 90 (2014) 015009 [arXiv: 1404.2924] [INSPIRE].

[27] J. Liu, N. McGinnis, C.E.M. Wagner and X.-P. Wang, Searching for the Higgsino-Bino Sector at the LHC, JHEP 09 (2020) 073 [arXiv: 2006. 07389] [INSPIRE].

[28] T. Han, S. Padhi and S. Su, Electroweakinos in the light of the Higgs boson, Phys. Rev. D 88 (2013) 115010 [arXiv:1309.5966] [INSPIRE]. 
[29] S.Y. Choi and Y.G. Kim, Analysis of the neutralino system in two body decays of neutralinos, Phys. Rev. D 69 (2004) 015011 [hep-ph/0311037] [INSPIRE].

[30] A. Canepa, T. Han and X. Wang, The search for electroweakinos, arXiv:2003.05450 [INSPIRE].

[31] T. Aoyama et al., The anomalous magnetic moment of the muon in the Standard Model, Phys. Rept. 887 (2020) 1 [arXiv: 2006. 04822] [INSPIRE].

[32] RBC, UKQCD collaboration, Calculation of the hadronic vacuum polarization contribution to the muon anomalous magnetic moment, Phys. Rev. Lett. 121 (2018) 022003 [arXiv: 1801.07224] [INSPIRE].

[33] Muon G-2 collaboration, Final report of the muon E821 anomalous magnetic moment measurement at BNL, Phys. Rev. D 73 (2006) 072003 [hep-ex/0602035] [InSPIRE].

[34] J.R. Ellis, J.S. Hagelin and D.V. Nanopoulos, Spin 0 leptons and the anomalous magnetic moment of the muon, Phys. Lett. B 116 (1982) 283 [INSPIRE].

[35] T. Moroi, The muon anomalous magnetic dipole moment in the minimal supersymmetric standard model, Phys. Rev. D 53 (1996) 6565 [Erratum ibid. 56 (1997) 4424] [hep-ph/9512396] [INSPIRE].

[36] M. Carena, G.F. Giudice and C.E.M. Wagner, Constraints on supersymmetric models from the muon anomalous magnetic moment, Phys. Lett. B 390 (1997) 234 [hep-ph/9610233] [INSPIRE].

[37] A. Czarnecki and W.J. Marciano, The muon anomalous magnetic moment: a harbinger for 'new physics', Phys. Rev. D 64 (2001) 013014 [hep-ph/0102122] [INSPIRE].

[38] J.L. Feng and K.T. Matchev, Supersymmetry and the anomalous magnetic moment of the muon, Phys. Rev. Lett. 86 (2001) 3480 [hep-ph/0102146] [INSPIRE].

[39] S.P. Martin and J.D. Wells, Muon anomalous magnetic dipole moment in supersymmetric theories, Phys. Rev. D 64 (2001) 035003 [hep-ph/0103067] [INSPIRE].

[40] J.S. Lee et al., CPsuperH: a computational tool for Higgs phenomenology in the minimal supersymmetric standard model with explicit CP-violation, Comput. Phys. Commun. 156 (2004) 283 [hep-ph/0307377] [INSPIRE].

[41] J.S. Lee, M. Carena, J. Ellis, A. Pilaftsis and C.E.M. Wagner, CPsuperH2.3: an updated tool for phenomenology in the MSSM with explicit CP-violation, Comput. Phys. Commun. 184 (2013) 1220 [arXiv: 1208.2212] [INSPIRE].

[42] M. Drees and M.M. Nojiri, The neutralino relic density in minimal $N=1$ supergravity, Phys. Rev. D 47 (1993) 376 [hep-ph/9207234] [INSPIRE].

[43] J.R. Ellis, T. Falk, K.A. Olive and M. Srednicki, Calculations of neutralino-stau coannihilation channels and the cosmologically relevant region of MSSM parameter space, Astropart. Phys. 13 (2000) 181 [Erratum ibid. 15 (2001) 413] [hep-ph/9905481] [INSPIRE].

[44] T. Han, F. Kling, S. Su and Y. Wu, Unblinding the dark matter blind spots, JHEP 02 (2017) 057 [arXiv: 1612 . 02387] [INSPIRE].

[45] M. Abdughani, L. Wu and J.M. Yang, Status and prospects of light bino-higgsino dark matter in natural SUSY, Eur. Phys. J. C 78 (2018) 4 [arXiv:1705.09164] [INSPIRE].

[46] G.H. Duan, W. Wang, L. Wu, J.M. Yang and J. Zhao, Probing GeV-scale MSSM neutralino dark matter in collider and direct detection experiments, Phys. Lett. B 778 (2018) 296 [arXiv: 1711.03893] [INSPIRE]. 
[47] M. Carena, J. Osborne, N.R. Shah and C.E.M. Wagner, Supersymmetry and LHC missing energy signals, Phys. Rev. D 98 (2018) 115010 [arXiv:1809.11082] [InSPIRE].

[48] M. Carena, J. Osborne, N.R. Shah and C.E.M. Wagner, Return of the WIMP: missing energy signals and the Galactic Center excess, Phys. Rev. D 100 (2019) 055002 [arXiv: 1905.03768] [INSPIRE].

[49] J. Cao, L. Meng, Y. Yue, H. Zhou and P. Zhu, Suppressing the scattering of WIMP dark matter and nucleons in supersymmetric theories, Phys. Rev. D 101 (2020) 075003 [arXiv: 1910.14317] [INSPIRE].

[50] ATLAS collaboration, Search for heavy Higgs bosons decaying into two tau leptons with the ATLAS detector using pp collisions at $\sqrt{s}=13$ TeV, Phys. Rev. Lett. 125 (2020) 051801 [arXiv: 2002.12223] [INSPIRE].

[51] CMS collaboration, Search for additional neutral MSSM Higgs bosons in the $\tau \tau$ final state in proton-proton collisions at $\sqrt{s}=13 \mathrm{TeV}, \mathrm{JHEP} 09$ (2018) 007 [arXiv:1803.06553] [INSPIRE].

[52] G. Bélanger, F. Boudjema, A. Pukhov and A. Semenov, MicrOMEGAs: a tool for dark matter studies, Nuovo Cim. C 033N2 (2010) 111 [arXiv:1005.4133] [INSPIRE].

[53] P. Draper, G. Lee and C.E.M. Wagner, Precise estimates of the Higgs mass in heavy supersymmetry, Phys. Rev. D 89 (2014) 055023 [arXiv: 1312.5743] [INSPIRE].

[54] E. Bagnaschi, G.F. Giudice, P. Slavich and A. Strumia, Higgs mass and unnatural supersymmetry, JHEP 09 (2014) 092 [arXiv:1407.4081] [INSPIRE].

[55] J. Pardo Vega and G. Villadoro, SusyHD: Higgs mass determination in supersymmetry, JHEP 07 (2015) 159 [arXiv: 1504.05200] [INSPIRE].

[56] G. Lee and C.E.M. Wagner, Higgs bosons in heavy supersymmetry with an intermediate $m_{A}$, Phys. Rev. D 92 (2015) 075032 [arXiv:1508.00576] [INSPIRE].

[57] H. Bahl, S. Heinemeyer, W. Hollik and G. Weiglein, Reconciling EFT and hybrid calculations of the light MSSM Higgs-boson mass, Eur. Phys. J. C 78 (2018) 57 [arXiv:1706.00346] [INSPIRE].

[58] H. Bahl et al., Precision calculations in the MSSM Higgs-boson sector with FeynHiggs 2.14, Comput. Phys. Commun. 249 (2020) 107099 [arXiv:1811.09073] [INSPIRE].

[59] H. Bahl and W. Hollik, Precise prediction for the light MSSM Higgs boson mass combining effective field theory and fixed-order calculations, Eur. Phys. J. C 76 (2016) 499 [arXiv: 1608.01880] [INSPIRE].

[60] T. Hahn, S. Heinemeyer, W. Hollik, H. Rzehak and G. Weiglein, High-precision predictions for the light CP-even Higgs boson mass of the minimal supersymmetric standard model, Phys. Rev. Lett. 112 (2014) 141801 [arXiv:1312.4937] [InSPIRE].

[61] W. Hollik and S. Paßehr, Higgs boson masses and mixings in the complex MSSM with two-loop top-Yukawa-coupling corrections, JHEP 10 (2014) 171 [arXiv:1409.1687] [INSPIRE].

[62] G. Degrassi, S. Heinemeyer, W. Hollik, P. Slavich and G. Weiglein, Towards high precision predictions for the MSSM Higgs sector, Eur. Phys. J. C 28 (2003) 133 [hep-ph/0212020] [INSPIRE].

[63] S. Heinemeyer, W. Hollik and G. Weiglein, The masses of the neutral CP-even Higgs bosons in the MSSM: Accurate analysis at the two loop level, Eur. Phys. J. C 9 (1999) 343 [hep-ph/9812472] [INSPIRE]. 
[64] S. Heinemeyer, W. Hollik and G. Weiglein, FeynHiggs: a program for the calculation of the masses of the neutral CP even Higgs bosons in the MSSM, Comput. Phys. Commun. 124 (2000) 76 [hep-ph/9812320] [INSPIRE].

[65] A. Djouadi, M.M. Muhlleitner and M. Spira, Decays of supersymmetric particles: The Program SUSY-HIT (SUspect-SdecaY-HDECAY-InTerface), Acta Phys. Polon. B 38 (2007) 635 [hep-ph/0609292] [INSPIRE].

[66] W. Beenakker, R. Hopker and M. Spira, PROSPINO: a program for the production of supersymmetric particles in next-to-leading order QCD, hep-ph/9611232 [INSPIRE].

[67] J.D. Wells, Implications of supersymmetry breaking with a little hierarchy between gauginos and scalars, in the proceedings of the $11^{\text {th }}$ International Conference on Supersymmetry and the Unification of Fundamental Interactions (SUSY 2003), June 5-10, Tucson, U.S.A. (2003), hep-ph/0306127 [INSPIRE].

[68] N. Arkani-Hamed and S. Dimopoulos, Supersymmetric unification without low energy supersymmetry and signatures for fine-tuning at the LHC, JHEP 06 (2005) 073 [hep-th/0405159] [INSPIRE].

[69] G.F. Giudice and A. Romanino, Split supersymmetry, Nucl. Phys. B 699 (2004) 65 [Erratum ibid. 706 (2005) 487] [hep-ph/0406088] [INSPIRE].

[70] B. Dutta, Y. Gao and B. Shakya, Light Higgsino decays as a probe of the NMSSM, Phys. Rev. D 91 (2015) 035016 [arXiv:1412.2774] [INSPIRE].

[71] T. Han, Z. Liu and S. Su, Light neutralino dark matter: direct/indirect detection and collider searches, JHEP 08 (2014) 093 [arXiv: 1406.1181] [INSPIRE].

[72] S. Baum, N.R. Shah and K. Freese, The NMSSM is within Reach of the LHC: mass correlations\& decay signatures, JHEP 04 (2019) 011 [arXiv: 1901.02332] [INSPIRE].

[73] R.K. Barman, G. Bélanger, B. Bhattacherjee, R. Godbole, D. Sengupta and X. Tata, Current bounds and future prospects of light neutralino dark matter in NMSSM, arXiv:2006.07854 [INSPIRE]. 